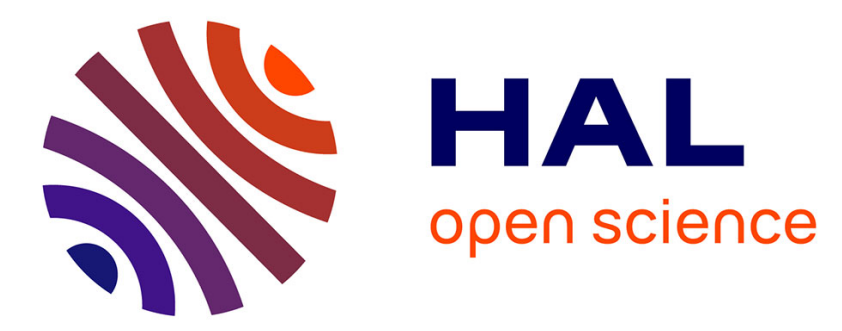

\title{
Histoire du temps présent et politique en Pologne
}

Valentin Behr

\section{To cite this version:}

Valentin Behr. Histoire du temps présent et politique en Pologne. Les Cahiers Sirice / Irice , 2018. halshs-02077658

\section{HAL Id: halshs-02077658 \\ https://shs.hal.science/halshs-02077658}

Submitted on 23 Mar 2019

HAL is a multi-disciplinary open access archive for the deposit and dissemination of scientific research documents, whether they are published or not. The documents may come from teaching and research institutions in France or abroad, or from public or private research centers.
L'archive ouverte pluridisciplinaire HAL, est destinée au dépôt et à la diffusion de documents scientifiques de niveau recherche, publiés ou non, émanant des établissements d'enseignement et de recherche français ou étrangers, des laboratoires publics ou privés. 
Cet article est paru dans Les Cahiers SIRICE, vol. 2, n 21, 2018, p. 121-137.

\section{Histoire du temps présent et politique en Pologne}

La Pologne a connu et continue de connaître, depuis le début des années 2000, des débats récurrents concernant son histoire récente. Est notamment en cause la gestion du passé communiste et l'héritage de la République populaire polonaise (PRL, 1944-1989), avec des interrogations sur le devenir des serviteurs de l'ancien-régime (lustration) et le sens des transformations de 1989 et de la «transition démocratique ${ }^{1}$. Les relations polono-juives et l'éventuelle coresponsabilité polonaise dans l'Holocauste constituent un autre point d'achoppement, dont les controverses autour des ouvrages de Jan T. Gross ont donné plusieurs illustrations ${ }^{2}$. A ces débats s'ajoute la création, en 1999, de l'Institut de la mémoire nationale (IPN), qui abrite le principal centre de recherches en histoire du temps présent.

Ces débats récurrents sur l'histoire récente et ses usages politiques ont fait l'objet de l'attention des historiens polonais, qui se sont interrogés en particulier sur le sens de la notion de «politique historique ${ }^{3}$ », variante des «politiques mémorielles ${ }^{4} »$. Il serait cependant réducteur de n'attribuer à ces historiens qu'un rôle d'analystes de débats sur le passé dont ils sont largement acteurs, tout au moins pour ceux d'entre eux qui se spécialisent en histoire du temps présent (historia najnowsza), celle de la période 1939-1989, qui correspond à l'expérience polonaise des deux «totalitarismes ${ }^{5}$ », nazi et soviétique. Cette participation des historiens, en tant que savants, aux débats sur le passé se donne à voir dans la pratique, fréquente chez les contemporanéistes, du «publicisme»(publicystyka), qui consiste à intervenir dans les médias (la presse, le plus souvent) pour y défendre telle ou telle interprétation. Ces interventions font parfois l'objet de publications sous forme de recueils ${ }^{6}$. Sur le plan éditorial, la production savante (ou semi-savante) des contemporanéistes polonais s'adresse donc aussi bien à un circuit de diffusion restreint (monographies et articles scientifiques) qu'à un circuit de diffusion large (vulgarisation, essayisme), pour reprendre les catégories proposées par Pierre Bourdieu ${ }^{7}$. Cette situation n'est pas spécifique à la Pologne,

\footnotetext{
${ }^{1}$ Jérôme Heurtaux et Frédéric Zalewski, Introduction à l'Europe postcommuniste, Bruxelles, De Boeck, 2012 , en particulier la première partie.

${ }^{2}$ Jan Tomasz Gross, Les voisins : 10 juillet 1941, un massacre de Juifs en Pologne, Paris, Fayard, 2002 ; Jan Tomasz Gross, La peur : l'antisémitisme en Pologne après Auschwitz, Paris, Calmann-Lévy, 2010 ; Audrey Kichelewski, «La peur des Juifs ou des Juifs qui ont peur? », Annales. Histoire, Sciences Sociales, 2009, $\mathrm{n}^{\circ} 5$, p. $1091-1104$.

3 Sławomir M. Nowinowski, Jan Pomorski et Rafał Stobiecki (dir.), Pamięć i polityka historyczna: doświadczenia Polski $i$ jej sąsiadów, Łódź, IPN, 2008 ; Paweł Skibiński, Tomasz Wiścicki et Michał Wysocki (dir.), Historycy i politycy: polityka pamięci w III RP, Warszawa, Wydawnictwo DiG, 2011. Pour un aperçu des débats entourant cette notion, promue par le gouvernement PiS (Droit et Justice) lors de sa première accession au pouvoir en 2005, voir Valentin Behr, " Genèse et usages d'une politique publique de l'histoire », Revue d'études comparatives Est-Ouest, 2015, vol. 46, nº 3, p. 21-48.

${ }^{4}$ Sarah Gensburger, Les Justes de France: politiques publiques de la mémoire, Paris, Presses de Sciences Po, 2010 ; Sarah Gensburger et Agnieszka Niewiedzial, «Figure du Juste et politique publique de la mémoire en Pologne (1945-2005) », Critique internationale, 2007, vol. 1, n 34, p. 127-148.

${ }^{5}$ Nous plaçons cette notion entre guillemets car nous ne la reprenons pas à notre compte. Sa dimension normative et sa faible pertinence pour rendre intelligibles les rapports de domination politique dans les régimes dits totalitaires a fait l'objet de nombreux commentaires. Voire en particulier Sandrine Kott, « Pour une histoire sociale du pouvoir en Europe communiste », Revue d'histoire moderne et contemporaine, 2002, vol. 49, $\mathrm{n}^{\circ} 2$, p. 5-23.

6 Entre autres: Marcin Kula, Naród, historia i... dużo kłopotów, Cracovie, Universitas, 2011 ; Paweł Machcewicz, Spory o historię 2000-2011, Kraków, Wydawnictwo Znak, 2012 ; Andrzej Nowak, Strachy $i$ Lachy: przemiany polskiej pamięci 1982-2012, Cracovie, Biały Kruk, 2012.

${ }^{7}$ Pierre Bourdieu, Homo academicus, Paris, Les éditions de Minuit, 1984.
} 
l'histoire pouvant s'appuyer sur la demande d'un lectorat profane, peut-être plus étendu dans le cas de cette discipline que pour d'autres disciplines des sciences sociales. La particularité polonaise, de ce point de vue, réside plutôt dans le fait que le débat historiographique s'y déroule davantage dans la presse généraliste, qui réunit régulièrement des panels d'historiens afin de débattre des sujets qui font polémique, que dans les revues scientifiques. De plus, la contribution des historiens à la fabrique de l'histoire ne se borne pas à la construction de représentations du passé. Elle passe aussi par le concours apporté par certains d'entre eux à de véritables politiques publiques ayant pour objet la mise en récit du passé. La «politique historique » menée par les gouvernements successifs a ainsi eu pour conséquence d'offrir aux historiens des débouchés professionnels attractifs, en-dehors du monde académique stricto sensu : l'IPN déjà cité, les musées, les archives nationales, les administrations en charge de la lustration, les cabinets ministériels, rendent possibles des carrières à l'intersection de l'académie et de la haute fonction publique ou de l'expertise.

Par conséquent, comprendre ce qui se joue à travers les controverses historiographiques et le rôle qu'y tiennent les historiens, implique de ne pas appréhender ces derniers seulement comme des chercheurs et des professionnels de l'histoire, ce qu'ils sont et ce qu'ils revendiquent comme étant leur identité professionnelle, mais aussi comme des acteurs des luttes politiques pour la définition du récit historique légitime, ce qu'ils peuvent être également, bien qu'ils soient moins prompts à le revendiquer. En interrogeant les frontières entre science et politique, il s'agit de fournir ici des éléments montrant comment la pratique de l'histoire du temps présent, dans le contexte polonais, oscille entre science de gouvernement ${ }^{8}$ et engagement intellectuel ${ }^{9}$; entre expertise et contribution à la production de visions du monde, d'idées politiques. On s'appuie pour ce faire sur les approches en termes de champ, qui semblent particulièrement heuristiques pour décrire les espaces de production des biens symboliques ${ }^{10}$.

\section{1/ Une sociologie politique de la fabrique de l'histoire}

Les usages politiques du passé et l'émergence des enjeux mémoriels dans l'espace public ont généralement été associés à l'effacement ou à la transformation du métier d'historien. L'idée d'une «dépossession» des historiens du monopole de l'interprétation du passé, au profit d'acteurs profanes (journalistes, " témoins », « victimes », «politiques », etc.), « comme si des étrangers étaient arrivés à l'improviste dans une corporation en changeant sans crier gare ses règles de fonctionnement », a été par exemple clairement exprimée par François Hartog et Jacques Revel :

"Des questions qui avaient été jusque-là l'objet de débats internes à la profession se trouvent aujourd'hui déférées devant l'opinion, transformant du même coup le statut de l'historien : soit qu'il devienne à son tour un enjeu, soit qu'on lui demande une expertise, soit qu'il prenne l'initiative d'intervenir dans le débat public, de le nourrir ou de l'initier. ${ }^{11}{ }$ ”

\footnotetext{
${ }^{8}$ Voir par analogie Franck Poupeau, Une sociologie d'État : l'école et ses experts en France, Paris, Raisons d'agir, 2003.

9 Gisèle Sapiro, «Modèles d'intervention politique des intellectuels », Actes de la recherche en sciences sociales, 2009, vol. 1, n 176-177, p. 8-31.

${ }^{10}$ Pierre Bourdieu, Les règles de l'art: genèse et structure du champ littéraire, Paris, Seuil, 1992 ; Gisèle Sapiro, La guerre des écrivains : 1940-1953, Paris, Fayard, 1999.

${ }^{11}$ François Hartog et Jacques Revel (dir.), Les usages politiques du passé, Paris, Éditions de l'EHESS, 2001.
} 
Les historiens polonais ne sont pas en reste lorsqu'il s'agit d'attribuer aux profanes, politiciens ou journalistes, la responsabilité des polémiques ayant le passé pour objet ${ }^{12}$. Pourtant, les historiens sont partie prenante des 《 usages publics de l'histoire ${ }^{13}$. Ceci ne doit pas surprendre, dans la mesure où «tout travail historique véhicule aussi, implicitement, un jugement sur le passé ${ }^{14} »$. Cependant, l'histoire et les historiens apparaissent rarement dans les recherches sur les engagements intellectuels ou le rôle politique des sciences (sociales). Comme si cette discipline ne pouvait jouer qu'un rôle marginal en tant que science de gouvernement ${ }^{15}$ (contrairement au droit ou à l'économie) et comme si elle concourait moins à la production des idées politiques (contrairement à la philosophie). Si plusieurs travaux ont été consacrés au rôle social et politique des historiens ${ }^{16}$, nous entendons étudier la contribution de l'histoire à la construction sociale de la réalité en nous appuyant également sur un ensemble de travaux consacrés au rôle politique joué par d'autres disciplines, ainsi qu'aux engagements intellectuels ${ }^{17}$.

L'approche en termes de champ, appliquée par Pierre Bourdieu et d'autres auteurs à l'étude de la production des biens symboliques, permet d'interroger l'autonomie, aussi bien que l'hétéronomie, du métier d'historien ou, plus précisément, de la fabrique du récit historique ${ }^{18}$. Loin d'être simplement victimes d'une dépossession de leur objet de recherche, les historiens (ou du moins une partie d'entre eux) contribuent et participent à des usages de la science historique qui ne se résument pas à un simple objectif de connaissance du passé, mais peuvent contribuer à ériger le savoir historien en ressource politique. Le savoir sur le passé est alors invoqué pour légitimer/délégitimer une prise de position politique dans le présent, ou bien pour délimiter le champ du pensable politiquement. En ce sens, la politique de l'histoire ne se comprend pas uniquement comme l'ingérence des pouvoirs publics dans la recherche savante ; c'est aussi la politique des historiens, la politique par d'autres moyens. Une telle observation plaide pour une étude approfondie de la structuration de l'espace de production historiographique, afin de comprendre quelles sont les oppositions et les luttes qui le structurent.

Outre les débats mentionnés en introduction, l'historiographie polonaise de la période 19391989 a connu un profond renouvellement au cours des années 2000. La spécialisation d'un groupe d'historiens dans l'étude de l'histoire nationale au cours de la Seconde Guerre

\footnotetext{
12 Voir par exemple l'entretien croisé qui ouvre le livre de Paweł Skibiński, Tomasz Wiścicki et Michał Wysocki (dir.), Historycy i politycy: polityka pamięci $w$ III RP, op. cit.

${ }^{13}$ Gérard Noiriel, « De quelques usages publics de l'histoire », Tracés. Revue de Sciences humaines, 2009, $\mathrm{n}^{\circ} 9$, p. $123-132$.

${ }^{14}$ Enzo Traverso, Le passé, modes d'emploi : histoire, mémoire, politique, Paris, La Fabrique, 2005.

15 Olivier Ihl, Martine Kaluszynski et Gilles Pollet (dir.), Les sciences de gouvernement, Paris, Economica, 2003 ; Olivier Ihl, Yves Déloye et Alfredo Joignant (dir.), Gouverner par la science : perspectives comparées, Grenoble, Presses universitaires de Grenoble, 2013.

${ }^{16}$ On peut citer, entre autres : Marie-Claire Lavabre et Dominique Damamme, « Les historiens dans l'espace public », Sociétés contemporaines, 2000, vol. 39, n 1, p. 5-21; Thomas Ribémont, L'expertise historienne dans la France contemporaine : la fonction politique de l'histoire en question, Thèse de doctorat en science politique, université Paris Dauphine, 2006 ; Olivier Dumoulin, Le rôle social de l'historien: de la chaire au prétoire, Paris, Albin Michel, 2003, 343 p ; Gérard Noiriel, Sur la « crise » de l'histoire, Paris, Belin, 1996.

${ }^{17}$ F. Poupeau, Une sociologie d'État, op. cit. ; Michel Amiot, Contre l'État, les sociologues : éléments pour une histoire de la sociologie urbaine en France, 1900-1980, Paris, Editions de l'EHESS, 1986 ; Frédérique Matonti, Intellectuels communistes: essai sur l'obéissance politique: la Nouvelle critique (1967-1980), Paris, La Découverte, 2005; Florent Champy et Liora Israël, «Professions et engagement public », Sociétés contemporaines, 2009, vol. 73, $\mathrm{n}^{\circ} 1$, p. 7-20.

${ }^{18}$ Voir notamment : P. Bourdieu, Les règles de l'art, op. cit. ; Frédéric Lebaron, Les économistes français entre science et politique: contribution à une sociologie de la culture économique, Thèse de doctorat, , thèse de doctorat en sociologie, EHESS, 1996 ; G. Sapiro, La guerre des écrivains, op. cit.
} 
mondiale et de la période communiste remonte cependant à l'immédiat après-guerre. Sans entrer ici dans de longs développements, il est utile de revenir sur la structuration de l'histoire du temps présent en Pologne, dont l'autonomie est un enjeu dès les origines ${ }^{19}$.

L'histoire, en particulier l'histoire contemporaine, fait comme la plupart des autres sciences humaines et sociales l'objet d'un strict contrôle idéologique en Pologne populaire. Censure, propagande, création de lieux de production d'une historiographie «officielle » (tel que le Département d'histoire du Parti), constituent autant de limites au développement d'une science historique autonome ${ }^{20}$. L'histoire de la Seconde Guerre mondiale et du régime communiste lui-même, essentielle à la légitimation du pouvoir, est indéniablement la plus soumise à la contrainte exercée par le Parti-État. Elle se développe néanmoins dès les années 1960-1970 au sein de l'Institut d'histoire de l'Académie des sciences, via la formation de petites équipes de chercheurs, souvent membres du Parti, dont les recherches évitent soigneusement l'histoire politique et se consacrent à des problématiques économiques, démographiques et sociales.

La donne change dans les années 1980 : en 1976 est fondé le KOR, Comité de défense des ouvriers, qui regroupe des universitaires et intellectuels opposés à la censure ; en août 1980, les accords de Gdansk légalisent le syndicat Solidarnosc. La dissidence constitue un réseau de diffusion de publications clandestines, dont une partie des contenus donne à voir une histoire non-censurée : des thèmes tels que l'exécution de prisonniers de guerre polonais par le NKVD à Katyn, en 1940, l'invasion soviétique du 17 septembre 1939, l'Insurrection de Varsovie ou encore les différentes révoltes anti-communistes (1956, 1968, 1970, 1976), jusqu'ici exclus ou travestis dans l'historiographie officielle, y sont abondamment traités. Certains historiens peuvent ainsi publier, sous pseudonyme et via les samizdats ou les maisons d'édition clandestines, les premiers travaux d'histoire politique portant sur la genèse de la République populaire de Pologne (PRL) ${ }^{21}$.

Cette contre-histoire née dans la dissidence devient dominante à la faveur de la «transition démocratique » amorcée en 1989. Dès le début des années 1990, l'historiographie de la période 1939-1989 entend offrir une «histoire vraie ${ }^{22}$ », libérée de la censure et de l'idéologie. Si elle a le grand mérite de combler les «taches blanches » héritées de l'ancienrégime, cette nouvelle historiographie véhicule aussi de nouvelles représentations politiques, à partir d'une posture de dévoilement et de dénonciation : la Pologne populaire est disqualifiée en tant que régime «totalitaire », «criminel » voire «étranger» car imposé par l'envahisseur soviétique, profondément inadapté à la société polonaise qui l'aurait massivement rejeté. L'histoire de la Pologne entre 1939 et 1989 serait celle d'une nation luttant pour son indépendance contre deux régimes, nazi et soviétique, dont les crimes envers la nation polonaise seraient équivalents.

Le paradigme «totalitaire » devient donc dominant dans l'historiographie polonaise post1989 et se trouve encore renforcé depuis la création de l'Institut de la mémoire nationale (IPN). Ce dernier conserve les archives des services de sécurité de la PRL, lesquelles sont prioritairement exploitées par plus de 200 historiens employés à l'Institut, qui est rapidement

\footnotetext{
${ }^{19}$ J'étudie la genèse de l'histoire du temps présent en Pologne dans ma thèse : Valentin Behr, « Science du passé et politique du présent en Pologne. L'histoire du temps présent (1939-1989), de la genèse à l'Institut de la mémoire nationale », thèse de doctorat en science politique, Université de Strasbourg, 2017.

${ }^{20}$ Rafał Stobiecki, Historiografia PRL: ani dobra, ani madra, ani piękna... ale skomplikowana, Varsovie, TRIO, 2007 ; Zbigniew Romek, Cenzura a nauka historyczna w Polsce 1944-1970, Varsovie, Wydawnictwo Neriton, 2010.

${ }^{21}$ Voir par exemple Andrzej Albert (pseudonyme de Wojciech Roszkowski), Najnowsza historia Polski 19181980, 1983 ; Krystyna Kersten, Narodziny systemu władzy, Polska 1943-1948 [Naissance du système de pouvoir 1943-1948], Poznań, SAWW, 1990.

${ }^{22}$ Rafał Stobiecki, «Reaktualizacja mitu historii „prawdziwej” w historiografii polskiej po 1989 r.» dans Pamiętnik XVI Powszechnego Zjazdu Historyków we Wrocławiu, Toruń, Adam Marszałek, 2002, p. 11-23.
} 
devenu un éditeur majeur d'ouvrages historiques ${ }^{23}$. Les recherches menées à partir de ces archives policières et militaires permettent de compléter la connaissance de l'ancien-régime, mais ont aussi pour conséquence d'en proposer une vision en noir et blanc ${ }^{24}$. Schématiquement, l'historiographie polonaise de la PRL oppose aujourd'hui, d'un côté, une histoire politique (ou totalitaire) dont le paradigme interprétatif repose sur l'opposition Etat/société. La PRL est alors analysée principalement en termes de «héros » et de "victimes », d'opposition démocratique et de répression étatique. L'IPN est aujourd'hui le lieu principal de production de ce type de récit. De l'autre côté, une histoire sociale qui s'intéresse à la vie quotidienne sous le communisme (vie des ouvriers, des femmes, des «gens ordinaires » ...) ou bien à une histoire sociale du pouvoir. Elle est essentiellement pratiquée dans les universités (en particulier celle de Varsovie) et à l'Académie des sciences. Cette opposition aboutit à la production d'interprétations divergentes de ce que fut la PRL ${ }^{25}$.

De cette genèse de l'histoire du temps présent en Pologne, rapidement esquissée, trois constats peuvent être tirés.

Premier constat : sur le plan de la pratique de l'histoire, la mise à distance des travaux de recherche réalisés à l'époque de la Pologne populaire, affublés du stigmate de science partisane, s'est traduite non seulement par le rejet du marxisme, mais aussi plus largement par la mise à distance de la réflexion conceptuelle. La majorité des spécialistes d'histoire du temps présent se réclame d'une approche factuelle et positiviste, reposant sur la collecte d'un grand nombre de sources (dont les contemporanéistes polonais ont longtemps été privés du temps de la PRL), en faisant le plus souvent l'économie d'une problématisation ou d'une conceptualisation trop poussée. Andrzej Paczkowski, l'un des principaux spécialistes de la période 1939-1989, revendique ainsi la pratique d'une histoire sans «problèmes »:

"Par bonheur, je pratique une histoire factuelle. Et même si je reconnais la pertinence de certaines définitions, je ne les utilise pas nécessairement. Je préfère montrer plutôt que qualifier. ${ }^{26}$ »

Ainsi que le font remarquer deux tenants d'une histoire sociale de la PRL, Marcin Zaremba et Błażej Brzostek, l'emploi de la notion de « totalitarisme » dans de nombreux travaux polonais relève davantage d'une déclaration de principe, par laquelle l'auteur indique sa désapprobation à l'égard de l'ancien-régime, que du recours à un paradigme durci ${ }^{27}$. Il est par conséquent de bon ton, parmi les spécialistes d'histoire du temps présent, de se démarquer de l'héritage de la période communiste, toute contribution qui tendrait à relativiser la dimension criminelle de l'ancien-régime ou à en souligner l'ancrage social risquant d'être assimilée à une tentative de réhabilitation.

Second constat : l'importance, dans le cas de l'histoire du nazisme et du communisme, de la commande étatique, qui agit comme une contrainte, externe, sur l'autonomie de la profession. Sans prétendre que cette commande soit de même nature ou d'intensité comparable entre la République populaire de Pologne et la Troisième République d'après 1989, des formes de

\footnotetext{
${ }^{23}$ Valentin Behr, «Historiens militants ou historiens de bureau? Les producteurs du récit historique officiel à l'Institut de la mémoire nationale », Revue d'études comparatives Est-Ouest, 2011, vol. 42, no 4, p. 5-35.

${ }^{24}$ Krzysztof Persak, «Mutations et usage public de l'historiographie polonaise de la seconde moitié du XXe siècle » dans Sonia Combe (dir.), Archives et histoire dans les sociétés post-communistes, Paris, La Découverte, 2009, p. 259-265.

${ }^{25}$ Pour davantage de précisions à ce sujet, voir Valentin Behr, « Historical policy-making in post-1989 Poland: a sociological approach to the narratives of communism », European Politics and Society, vol. 18, n ${ }^{\circ}$, 2017, p. 81-95.

${ }^{26}$ Cité in R. Stobiecki, «Reaktualizacja mitu historii „prawdziwej” w historiografii polskiej po 1989 r. [La réactualisation du mythe de l'histoire "vraie" dans l'historiographie polonaise après 1989] », art cit.

${ }^{27}$ Marcin Zaremba et Blażej Brzostek, «Polska 1956-1976: w poszukiwaniu paradygmatu », Pamięć $i$ Sprawiedliwość, 2006, vol. 10, nº 2, p. 25-37.
} 
continuité s'observent dans la volonté d'encourager la production et la diffusion d'un récit historique conforme aux attentes du pouvoir, via la mise en place d'institutions spécifiquement dédiées à la production d'un récit plus ou moins officiel : Département d'histoire du Parti, Institut de la mémoire nationale, commissions d'historiens. Le parallèle entre l'attention portée à l'histoire par ces deux régimes est d'ailleurs parfois établi par les acteurs eux-mêmes, récemment à l'occasion d'un «Forum des chercheurs en histoire contemporaine », consacré à la critique de la politique historique du gouvernement du parti Droit et Justice $(\mathrm{PiS})^{28}$. A travers la contrainte de la commande étatique, se donne donc à voir une conception de l'histoire en tant que science de gouvernement, l'historien mettant son expertise au service du pouvoir. C'est cette demande d'expertise qui est à l'origine de la création de débouchés extra-académiques, offrant ainsi d'autres types de carrières à ceux des professionnels de l'histoire qui acceptent de se prêter au jeu de l'expertise.

Troisième constat : à cette contrainte externe, qui remet en cause l'autonomie de la science historique, s'ajoute une contrainte interne, dont la nature est différente. L'engagement intellectuel des historiens, via leurs interventions dans le débat public, est un autre facteur de politisation du discours sur l'histoire. Comme d'autres intellectuels ${ }^{29}$, certains historiens font le choix de mettre à profit leur statut de savant pour prendre des positions politiques. Ils y sont d'autant plus incités dans un pays où les débats sur le passé récent sont, on l'a dit, récurrents, d'où une forte demande en ce sens, émanant des médias ou de la société civile. En délivrant des représentations du passé qui peuvent être politiquement et socialement utiles à une cause, les historiens sont eux-mêmes acteurs de la politisation de l'histoire. Cette politisation relativise certes l'autonomie de la discipline, mais elle constitue aussi une ressource, les engagements intellectuels pouvant permettre d'engranger des profits de notoriété, voire faciliter la nomination à un poste prestigieux. Il s'agit donc ici de nuancer la contrainte externe, en tenant compte des acquis de la sociologie des engagements intellectuels, et donc des conditions dans lesquelles l'histoire est produite.

En adoptant cette distinction entre contrainte externe et contrainte interne, il ne s'agit pas pour nous de rigidifier une distinction qui est faite ici avant tout pour la clarté du propos, mais d'exprimer le fait que deux types de tensions s'agencent pour restreindre l'autonomie de l'histoire savante. Les logiques de concurrence internes à l'espace de production historiographique consistent précisément à se positionner vis-à-vis des contraintes externes, qui peuvent tout aussi bien, lorsqu'elles sont réfractées dans cet espace ${ }^{30}$, constituer des ressources pour les acteurs qui se les approprient. L'articulation de ces deux types de contraintes permet de comprendre les logiques de l'engagement politique et intellectuel des historiens polonais du temps présent, dans des configurations aussi différentes que la période stalinienne du début des années 1950 et les années 2000-2010.

Il s'agit ainsi de montrer que les controverses historiographiques qui se donnent à voir aujourd'hui doivent peut-être moins aux effets (différés) de la rupture de 1989, qu'à un prolongement, sous des formes actualisées, des luttes qui structurent l'histoire du temps présent depuis sa genèse en Pologne populaire, dont l'enjeu principal est celui de l'autonomie de la discipline et de son rapport au politique.

\section{2/ Le rôle politique des historiens}

\footnotetext{
${ }^{28} \mathrm{La}$ conférence a été filmée et peut être vue ici : http://pth.net.pl/aktualnosci/200.

${ }^{29}$ Christophe Charle, Naissance des «intellectuels » : 1880-1900, Paris, Minuit, 1990, 271 p.

${ }^{30}$ Sur la notion de réfraction, voir P. Bourdieu, Les règles de l'art, op. cit., p. 360-361.
} 
Afin d'illustrer ce qui précède, il s'agit à présent d'exposer quelques modalités saillantes, mais non exhaustives, de l'engagement politique des historiens du temps présent en Pologne. En soulignant des trajectoires typiques d'individus qui occupent ou ont occupé une place centrale dans les débats historiographiques du moment, dont les parcours dérogent à la vision idéalisée d'universitaires autonomes, au-dessus des contingences du débat politique. Evoluant à la frontière entre science et politique, ils font de la politique en tant qu'historiens, au nom d'une compétence savante ou experte. Dès lors, les modalités de leur engagement politique relèvent plutôt de l'intervention d'intellectuels, selon le modèle esquissé par Gisèle Sapiro ${ }^{31}$. On a volontairement choisi de mettre l'accent ici sur des cas se rapprochant davantage de l'hétéronomie que de l'autonomie, afin d'illustrer et d'interroger la porosité des frontières entre science et politique, à travers quatre modalités d'engagement politique des historiens contemporanéistes polonais.

Un cas limite de la contrainte externe, exercée par l'Etat sur la recherche en histoire, se donne à voir dans le Département d'histoire du Parti (WHP), fondé en 1946 auprès du Comité central du Parti. Chargé de la production et de la vulgarisation d'une histoire officielle du «mouvement ouvrier », c'est-à-dire du Parti et des mouvements l'ayant précédé, le WHP incarne une histoire sur commande, hétéronome. Prenant ses instructions directement auprès du Comité central, voire du Premier secrétaire, il fonctionne jusqu'à sa suppression, en 1971, isolément du reste de la science historique, qui s'élabore dans les universités et à l'Académie des sciences. Y travaillent non pas des historiens professionnels, mais des militants du Parti, souvent dépourvus de formation universitaire. A l'image de Tadeusz Daniszewski, directeur du WHP de 1948 à 1968. Militant communiste dès les années 1920 (il adhère au Parti à 17 ans), il est nommé directeur de l'école du Parti en 1944, avant de prendre la tête du WHP. Il dirige parallèlement la chaire d'histoire du mouvement ouvrier à l'Institut de formation des cadres scientifiques, lui aussi rattaché au Comité central du Parti. Promu professeur en 1956 alors qu'il n'a pas de doctorat, il sera ensuite nommé à l'Institut d'histoire de l'Académie des sciences, où il se consacrera aux relations polono-soviétiques, autre thème à forte charge idéologique. A l'instar de Daniszewski, les employés du WHP sont pour l'essentiel des militants historiens. Nous reprenons, en la modifiant, cette expression de Georges Mink, qui qualifie d' " historiens militants » certains chercheurs de l'IPN, dont il sera fait mention plus bas $^{32}$. Si ceux qui sont ainsi qualifiés par G. Mink disposent des titres universitaires leur conférant le statut d'historiens professionnels, tel n'est pas le cas des militants historiens des années 1950 et 1960. Ces derniers sont des profanes disposant rarement d'une formation universitaire, arrivés sur le tard dans le monde académique, après avoir connu des carrières de militants professionnels au sein du Parti, où ils ont le plus souvent été en charge des activités intellectuelles, telles que la propagande ou la formation des militants. Le contexte de l'aprèsguerre est très favorable à ces nouveaux-venus dans le champ historien, qui de par leur expérience au service du Parti, sont les plus à mêmes de maîtriser la vulgate marxiste qui fournit alors le cadre conceptuel de la recherche historique. Pour ces intellectuels d'organisation qui doivent tout ce qu'ils sont à leur engagement militant, investir une carrière académique est également une opportunité de promotion sociale.

Tout autre est le cas des intellectuels dissidents, compagnons de route de Solidarnosc, où ils font office de conseillers. Parmi eux se trouvent de nombreux universitaires de carrière, qui passent en politique après 1989 . Si, parmi les historiens, les noms les plus connus sont ceux de médiévistes (Bronisław Geremek, ministre des Affaires étrangères entre 1997 et 2000 ;

\footnotetext{
${ }^{31}$ G. Sapiro, «Modèles d'intervention politique des intellectuels », art cit.

${ }^{32}$ Georges Mink, « Les usages des instituts de la mémoire nationale (I.M.N.) dans les recompositions partisanes (1989-2008) » dans Pascal Bonnard et Georges Mink (dir.), Le passé au présent, Paris, Michel Houdiard, 2011, p. 171-184.
} 
Karol Modzelewski, fondateur de l'Union du travail, un parti social-démocrate), il s'y trouve aussi quelques rares contemporanéistes. Władysław Bartoszewski est l'un d'eux. Arrêté à 18 ans lors d'une rafle, il est emprisonné au camp d'Auschwitz de 1940 à 1941, puis rejoint la résistance. Au sein de l'Armée de l'intérieur (AK), il s'occupe de l'aide apportée aux juifs en créant l'organisation Żegota. Bien qu'il ne soit pas historien de formation (il obtient un diplôme de philologie polonaise à l'université clandestine de Varsovie en 1944), son expérience d'ancien combattant lui vaut de faire office d'expert après-guerre. Il collabore ainsi aux travaux de la Commission centrale de recherche sur les crimes allemands en Pologne, avant de publier son ouvrage majeur, consacré à l'aide apportée par des Polonais aux juifs durant la guerre, paru en $1966^{33}$. Il consacre plusieurs autres ouvrages à l'histoire de la résistance et de l'Insurrection de Varsovie, à laquelle il a participé, dont certains sont publiés dans la clandestinité. Bartoszewski enseigne également l'histoire contemporaine, à l'Université catholique de Lublin, une université privée, affranchie du pouvoir, dans les années 1970 et 1980, puis dans diverses universités allemandes, en tant que professeur invité. A l'instar d'autres historiens proches de la dissidence, ses compétences lui valent d'être nommé ambassadeur en Autriche de 1990 à 1995. Il sera ensuite par deux fois ministre des Affaires étrangères en 1995 puis de 2000 à 2001, succédant à Geremek. De 2007 à sa mort, en 2015, il est enfin secrétaire d'Etat à la chancellerie du Premier ministre (Donald Tusk puis Ewa Kopacz, libéraux de la Plateforme civique), chargé du dialogue international. Son passage en politique ne l'empêche pas de continuer à publier des livres d'histoire et de présider le Conseil international du Musée d'Auschwitz-Birkenau (1990-2014). La trajectoire de Bartoszewski illustre donc bien celle d'intellectuels critiques, issus de la dissidence, passés en politique à la faveur des transformations initiées en 1989, en devenant conseillers du prince ou en occupant des fonctions politiques. Le phénomène ne s'est pas tari depuis : ces dernières années, plusieurs historiens siègent ou ont siégé à la Diète polonaise ou au Parlement européen.

Les experts, ou intellectuels d'institution, pour reprendre la formule suggérée par Gisèle Sapiro, offrent une troisième modalité d'engagement intellectuel. Les besoins engendrés par la politique historique ont ouvert aux historiens des carrières politico-administratives. Sławomir Cenckiewicz est un bon exemple de ceux que G. Mink qualifie d'historiens militants. Employé à l'IPN à partir de 2001, il obtient son doctorat en 2003 à l'université de Gdansk. Ce spécialiste des archives des services de sécurité de la Pologne populaire quitte brièvement l'IPN entre juillet et octobre 2006, après avoir été nommé président de la Commission de «liquidation» du renseignement militaire, établie par le premier gouvernement PiS (2005-2007). Cette commission a pour objectif de réorganiser le renseignement militaire et d'établir les actes contraires au droit imputables à ses membres, notamment des actes de collaboration avec les services de renseignement soviétiques et russes. Il travaille alors sous les ordres du vice-ministre de la Défense, Antoni Macierewicz, dont il est réputé proche. Nommé ministre de la Défense dans le gouvernement PiS formé suite aux élections de 2015, Macierewicz fait à nouveau appel à Cenckiewicz, à qui il confie la direction des archives militaires. La proximité idéologique de Cenckiewicz avec le PiS se donne également à voir dans le type d'histoire qu'il pratique : il est en effet le co-auteur d'un ouvrage à charge contre Lech Walesa, accusé de collaboration avec les services de sécurité communistes $^{34} \ldots$ mais aussi d'une biographie d'Anna Walentynowicz (intitulée Anna Solidarnosc), icône des grèves ouvrières de Gdansk en 1980, qui fait figure d'héroïne alternative à Walesa. Les recherches de Cenckiewicz, de même que sa focalisation sur les archives des services de sécurité, où résiderait la « vérité » sur l'ancien-régime, entrent donc

\footnotetext{
${ }^{33}$ Władysław Bartoszewski et Zofia Lewinówna, Ten jest z ojczyzny mojej: polacy z pomoca Żydom, 1939-1945, Cracovie, Znak, 1966.

${ }^{34}$ Sławomir Cenckiewicz et Piotr Gontarczyk, SB a Lech Watęsa: przyczynek do biografii, IPN., Gdańsk, 2008.
} 
en concordance avec l'entreprise de relecture de l'histoire récente de la Pologne menée par le PiS, qui entend remettre en question les accords de la Table ronde, conclus entre Solidarnosc et le Parti en $1989^{35}$.

Pawel Machcewicz défend pour sa part des interprétations historiques opposées à celles de Cenckiewicz, mais sa trajectoire est comparable. Il obtient son doctorat en 1993 à l'université de Varsovie, avant de rejoindre l'Institut d'études politiques de l'Académie des sciences, qui abrite un laboratoire spécialisé dans l'étude de la PRL. Ce co-fondateur de l'IPN en dirige le Bureau d'éducation publique (centre de recherches et d'éducation) de 2000 à 2006. Non reconduit dans ses fonctions par le gouvernement PiS, il intègre en 2008 le cabinet du Premier ministre Donald Tusk, où il est en charge de la conception du musée d'histoire de la seconde guerre mondiale à Gdansk, dont il est nommé directeur. L'exposition permanente du musée élaborée par Machcewicz et son équipe entend promouvoir une histoire "ouverte », présentant la guerre à travers les souffrances des populations civiles, polonaise mais aussi allemande et soviétique ... à l'opposé du récit nationalo-centré proposé par le PiS, dont le gouvernement cherche aujourd'hui à modifier cette exposition. L'érection de l'histoire en catégorie d'intervention publique ${ }^{36}$ à travers la «politique historique » semble donc avoir offert des opportunités de carrière à des historiens bureaucrates, assez nettement identifiés à l'un ou l'autre camp politique.

Enfin, une dernière modalité d'engagement intellectuel est le fait d'universitaires plus autonomes car moins engagés auprès de la commande étatique, qui pratiquent une intense activité de publicistes en intervenant régulièrement dans les médias. Andrzej Nowak en est un bon exemple. Professeur d'histoire rattaché à l'Académie des Sciences et à l'Université de Cracovie, ce spécialiste de l'histoire de l'Europe de l'Est et de la Russie aux $19^{\mathrm{e}}$ et $20^{\mathrm{e}}$ siècles est très présent dans les débats sur la politique historique et l'histoire du temps présent, bien qu'il ait lui-même fort peu travaillé sur cette période. Longtemps rédacteur en chef du bimensuel conservateur Arcana, considéré comme l'un des creusets intellectuels de la droite nationaliste, il publie fréquemment dans la presse conservatrice et catholique (Nasz Dziennik, Gazeta Polska, Gość Niedzielny). Fort de son statut de professeur d'histoire et de sa notoriété dans les milieux conservateurs proches du PiS, il est également devenu une figure de la promotion d'un grand récit national, notamment lorsqu'il fut érigé en porte-parole des opposants à une réforme des programmes d'enseignement de l'histoire promue par la Plateforme civique en 2012. Cette mobilisation a débouché sur l'inclusion dans les programmes scolaires d'un bloc thématique intitulé «Panthéon national », suggéré par Nowak. Egalement membre du conseil de la « Ligue polonaise anti-diffamation ${ }^{37}$ », qui veille à la façon dont l'histoire polonaise est traitée dans les médias étrangers, son engagement intellectuel lui a finalement permis de devenir conseiller du prince, puisqu'il a été nommé par le président Andrzej Duda (PiS), élu en 2015, dans l'équipe de conseillers en charge de la culture, de l'identité nationale et de la politique historique, ainsi qu'au Conseil de l'IPN.

A l'opposé, Adam Leszczynski, lui aussi spécialiste de l'histoire de la PRL, a été formé comme Machcewicz par le professeur de l'université de Varsovie Marcin Kula, tenant d'une histoire sociale de la Pologne populaire. Chercheur à l'Institut d'études politiques de l'Académie des sciences, il se consacre également au journalisme, spécialisé sur les thèmes historiques. Il collabore ainsi au quotidien Gazeta Wyborcza (résolument opposé à la politique historique du PiS), à la revue trimestrielle de gauche Krytyka Polityczna et plus récemment au média en ligne de fact checking Oko.press, très critique du gouvernement PiS.

\footnotetext{
${ }^{35}$ Frédéric Zalewski, «L'émergence d'une démocratie Antilibérale en Pologne », Revue d'études comparatives Est-Ouest, 2016, vol. 47, nº 4, p. 57-86.

${ }^{36}$ Voir par analogie Vincent Dubois, La politique culturelle: genèse d'une catégorie d'intervention publique, Paris, Belin, 2012.

${ }^{37}$ http://rdi.org.pl/.
} 
Ont été esquissées ici différentes modalités d'intervention politique des historiens. A travers leur production, polémique ou savante, la proximité qu'il entretiennent avec certains courants politiques ou encore les responsabilités qu'ils exercent au sein du monde politicoadministratif, ils prennent des positions qui sont à la fois savantes, renvoyant à une certaine pratique de l'histoire, et politiques, renvoyant à une certaine vision du monde. L'approche des débats historiographiques au prisme de la littérature consacrée aux engagements intellectuels permet de faire ressortir le rôle politique joué par les historiens dans ces débats, rôle qu'une opposition stricte entre le savant et le politique tendrait au contraire à minimiser. Si on a souligné ici quelques spécificités polonaises, le cadre d'analyse présenté ici pourrait s'appliquer à bien d'autres cas nationaux, comme le suggèrent certaines lectures du débat français sur les lois mémorielles ${ }^{38}$.

L'histoire du temps présent en Pologne apparaît éminemment nationale et relativement autonome vis-à-vis d'influences transnationales. Jan Grabowski, historien polonais spécialiste de la Shoah en poste à l'université d'Ottawa, a ainsi regrettée récemment l' "insularité » de cette histoire $^{39}$. Les historiens polonais du temps présent qui apparaissent aujourd'hui comme les plus internationalisés, à l'image de Małgorzata Mazurek, à la tête de la chaire d'histoire polonaise de l'université de Columbia, Robert Traba ou Maciej Górny, tous deux à l'antenne de l'Académie polonaise des sciences à Berlin, sont assez peu présents dans les débats historiographiques qui agitent régulièrement la profession. Ces historiens, souvent plus ouverts à une approche de l'histoire comme science sociale ouverte sur la sociologie ou l'anthropologie, sont emblématiques d'un courant historiographique aujourd'hui consacré à l'international, mais marginalisé en Pologne. L'historiographie dominante telle qu'elle se pratique à l'IPN, mais pas seulement, demeure caractérisée par culte de l'archive et de la factographie, accompagné de la mise à distance de la conceptualisation. Cette méthodologie puise ses sources dans l'héritage de la Pologne populaire et sa mise à distance en 1989, au nom d'une « histoire vraie » qui semble toujours constituer le programme de travail de bien des historiens du temps présent ${ }^{40}$. Le constat formulé par Pieter Lagrou résonne ainsi avec une acuité particulière dans le cas polonais :

"Quelle est dorénavant la cible d'une historiographie critique, quels en sont les contours géographiques? Ceux qui ont troqué l'histoire nationale pour l'histoire européenne, comparée, transnationale ou même globale ont aussi perdu au change leur lectorat (celui-là même qui transformait les historiens en intellectuels publics, si différents du peloton des spécialistes en sciences sociales aux objets abscons ou peu excitants pour les nonspécialistes). Ce que la nouvelle génération a gagné en couverture linguistique (accès aux sources, accès aux publications en langues étrangères, possibilité de publier, d'être lu et reconnu en dehors d'un espace national) a le plus souvent été payé par une perte d'impact dans des espaces publics encore largement nationaux. Apparaît le risque d'une séparation

\footnotetext{
38 Gérard Noiriel et Nicolas Offenstadt, «Histoire et politique autour d'un débat et de certains usages », Nouvelles FondationS, 2006, vol. 2, n 2, p. 65-75.

39 Jan Grabowski, «Miejsce Holokauste w polskiej polityce historycznej » [La place de l'Holocauste dans la

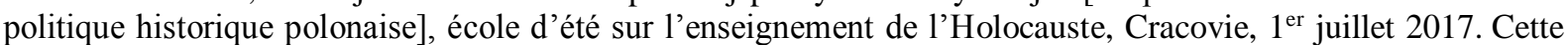
insularité nous semble toutefois devoir être relativisée par le travail de commissions bilatérales d'historiens, dont le rôle est certes de mettre l'histoire au service de politiques de réconciliation, mais qui contribuent, de par leurs publications, à produire un récit moins nationalo-centré. Voir notamment Anne Bazin, «La réconciliation à travers l'écriture d'une histoire commune: l'exemple des commissions d'historiens germano-tchèque et germano-polonaise », Revue d'études comparatives Est-Ouest, 2000, vol. 31, nº 1, p. 33-65; Andrzej Paczkowski, «Pologne et Ukraine. Questions délicates, réponses difficiles » dans Georges Mink et Laure Neumayer (dir.), L'Europe et ses passés douloureux, Paris, La Découverte, 2007, p. 143-155.

40 R. Stobiecki, «Reaktualizacja mitu historii „prawdziwej” w historiografii polskiej po 1989 r. [La réactualisation du mythe de l'histoire “vraie" dans l'historiographie polonaise après 1989] », art cit.
} 
radicale entre des historiens " globalisés » aux analyses innovantes et pertinentes pour leurs pairs mais ignorés d'un plus large public, et des historiens bouffons continuant d'abreuver éditeurs et publics nationaux adives de scoops, de tabous brisés et de projets historiographiques, dont la date de péremption est dépassée depuis des décennies ${ }^{41}$. »

${ }^{41}$ Pieter Lagrou, «De l'histoire du temps présent à l'histoire des autres », Vingtième Siècle, 2013, n ${ }^{\circ} 118$, p. 111-112. 